\title{
Analgésicos e antiinflamatórios na movimentação dentária induzida: metodologia e interpretação
}

\author{
Você sabe o que é extrapolação alométrica?*
}

\author{
Alberto Consolaro \\ Professor Titular em Patologia Bucal pela Faculdade de Odontologia de Bauru - USP
}

A falta de padronização nos procedimentos clínicos é generalizada, exceto em uma ou outra especialidade médica. A ausência de protocolos permite "opiniões, experiências pessoais, dicas, macetes e técnicas individuais", aplicadas amplamente nos pacientes, sem qualquer fundamentação na metodologia científica.

Por outro lado, o enrijecimento com base em protocolos muito bem definidos pode dificultar o tratamento de casos especiais e diferentes, fora da média. Ou melhor, pode dificultar a explicação, a justificativa e as conseqüências que podem advir de tratamentos fora do que está protocolado em uma sociedade, instituição científica ou profissional, inclusive do ponto de vista jurídico.

Em muitas áreas, os protocolos existem, mas por ignorância não se tem acesso ao conhecimento apropriado. Muitas vezes ficamos fechados em nosso assunto específico, sem nos preocuparmos em consultar outras áreas do conhecimento humano.

O tópico Material e Métodos nas teses e trabalhos publicados apresenta os protocolos experimentais utilizados, ou seja, os "métodos". Permeando o texto sobre a metodologia do trabalho, apresenta também os instrumentos, as drogas, os equipamentos e outros apetrechos utilizados durante os experimentos. $\mathrm{Na}$ língua portuguesa o substantivo coletivo para designar este conjunto de itens é "material". Esta palavra, quando utilizada como substantivo coletivo, não deve ser utilizada no plural, mas infelizmente alguns autores, revistas, teses e dissertações insistem em utilizar "materiais e métodos" ou "material e método" no lugar do correto Material e Métodos.

Apesar de poucas pessoas atentarem para a metodologia utilizada nos experimentos, sobre ela recai a maior parte da avaliação do trabalho a ser lido. Quando a metodologia tem limitações ou falhas, os trabalhos quase sempre são ignorados pela maioria dos estudiosos criteriosos do assunto ou são explicitamente criticados.

Nos trabalhos experimentais sobre os efeitos dos medicamentos na movimentação dentária induzida e nas reabsorções dentárias utilizam-se vários animais, sendo mais comuns os ratos, macacos e, mais esporadicamente, cães, gatos e porcos. Nos três últimos, as dificuldades técnicas, de padronização e de extrapolação dificultam a sua realização e a comparação de resultados.

$\mathrm{O}$ uso de animais em experimentos deve levar em consideração a taxa metabólica basal de cada organismo, mas infelizmente nem sempre isto é sabido e aplicado. A taxa de metabolismo basal refere-se à taxa metabólica determinada em condições padronizadas, em que o animal encontra-se quieto, sem digerir nenhum alimento, sem dormir, sem sofrer qualquer tipo de estresse e em temperatura ambiental ótima ${ }^{3}$. Considerada uma inter-relação fundamental que existe entre todos os seres vivos, a taxa metabólica basal é tida

\footnotetext{
* Agradeço a: 1) Prof. Dra. Rosangela Gonçalves Peccinini Machado, da Faculdade de Farmácia e Bioquímica da UNESP-Araraquara, pela gentileza e disponibilidade em discutir e fornecer dados para nos fundamentarmos apropriadamente sobre a Extrapolação Alométrica; 2) Ao doutorando Milton Santamaria Jr. pela colaboração recebida.
} 
como a melhor ferramenta para comparação entre organismos diferentes e utilizada por diversos pesquisadores para esta finalidade ${ }^{3}$. Em doses de medicamentos nos animais não se deve simplesmente aplicar a equação de dose por quilograma de peso, usada para o homem, extrapolando-a para o animal, pois a relação entre a taxa metabólica e a massa corporal não é linear.

Os organismos mantêm um intercâmbio contínuo de sustâncias com seu ambiente exterior: tomam as que precisam e cedem outras. Os organismos requerem, para sua manutenção, um fluxo contínuo de energia. O conjunto dos processos de troca com o ambiente e de obtenção de energia se denomina metabolismo e comumente nos referimos à taxa metabólica. Em estado de repouso o metabolismo basal no homem e nos animais aumenta na proporção do tamanho do corpo ${ }^{12}$. O incremento do metabolismo basal não parece estar em função direta do peso corporal: à medida que o peso corporal aumenta, diminui a troca energética por unidade de peso. O aumento do metabolismo parece depender muito mais da superfície corporal do que do peso ou massa corporal. Em cães com pesos corporais diferentes, Max Rubner (1883, apud PICHOTKA ${ }^{12}$, 1971) estabeleceu diferentes valores para a troca metabólica (Tab. 1), que ficaram conhecidos como "Lei de superfície corporal de Rubner" ${ }^{\prime \prime, 17}$. As mais variadas espécies animais também apresentam metabolismos diferentes (Tab. 2),

Tabela 1 - Troca metabólica em estado de repouso de 7 cães, quanto à unidade de peso e de superfície corporais, segundo Max Rubner (apud PICHOTKA $\left.{ }^{12}, 1971\right)$.

\begin{tabular}{|c|c|c|c|c|}
\hline$\underset{n^{0}}{\operatorname{animal}}$ & $\begin{array}{c}\text { peso em } \\
\text { quilogramas }\end{array}$ & $\begin{array}{c}\text { superfície } \\
\text { em } \mathbf{c m}^{2}\end{array}$ & $\begin{array}{c}\text { Kcal/Kg em } \\
24 \text { horas }\end{array}$ & $\begin{array}{c}\mathrm{Kcal} / \mathrm{m}^{2} \\
\text { de superfície }\end{array}$ \\
\hline 1 & 31,2 & 10.750 & 35,68 & 1.036 \\
\hline 2 & 24 & 8.805 & 40,91 & 1.112 \\
\hline 3 & 19,8 & 7.500 & 45,87 & 1.207 \\
\hline 4 & 18,2 & 7.662 & 46,20 & 1.097 \\
\hline 5 & 9,61 & 5.286 & 65,16 & 1.183 \\
\hline 6 & 6,5 & 3.724 & 66,07 & 1.153 \\
\hline 7 & 3,19 & 2.423 & 88,07 & 1.212 \\
\hline
\end{tabular}

conforme relatou Benedict ${ }^{2}$.

Nos ratos, por exemplo, o valor de troca metabólica do metabolismo basal (em Kcal $/ \mathrm{kg}$ ) é aproximadamente três vezes maior que no homem. Em raciocínio linear e direto, se assim fosse, a dose diária em murinos deveria ser triplicada em relação à dose humana por quilograma de peso.

Ao considerar-se a superfície corporal $\left(\mathrm{Kcal} / \mathrm{m}^{2}\right)$, 1,5 vezes menor, aproximadamente, a dose medicamentosa deveria ser um terço da dose humana, se aplicarmos experimentalmente o mesmo medicamento no rato, pela mesma via de administração.

No parâmetro Kcal/24h o valor para o homem é de 1.650 e do rato apenas 33,2 , ou seja, quase 50 vezes menor que o homem. $\mathrm{O}$ metabolismo basal também implica em velocidade. Suposta e teoricamente, no rato, o tempo entre as doses deveria ser reduzido a quase 50 vezes menos que o do homem, se considerarmos o valor de Kcal/24h, o que diminuiria em muito a dose diária em cada animal.

Diante destes supostos parâmetros surge um questionamento: em quantos trabalhos sobre medicamentos durante a movimentação dentária induzida em animais ${ }^{1,4,5,6,8,14,15,18,19,20}$ encontra-se explicitada esta preocupação com o índice metabólico específico de cada espécie, mesmo em suas críticas e análises em pareceres e julgamentos? Em quantos trabalhos ${ }^{1,4,5,6,8,14,15,18,19,20}$ foram explicitadas por que se utilizaram as doses referidas?

Alguns pesquisadores, consultores, avaliadores

Tabela 2 - Troca metabólica em estado de repouso de espécies distintas de animais quanto à unidade de peso e de superfície corporais, segundo Benedict (apud PICHOTKA ${ }^{12}$, 1971).

\begin{tabular}{ccccc}
\hline & $\begin{array}{c}\text { peso em } \\
\text { gramas }\end{array}$ & $\begin{array}{c}\text { Kcal/ } \\
\text { 24 horas }\end{array}$ & Kcal/Kg & $\begin{array}{c}\text { Kcal/m } \\
\text { de superfície }\end{array}$ \\
\hline camundongo & 21 & 3,6 & 158 & 550 \\
rato & 400 & 33,2 & 82 & 670 \\
coelho & 2.600 & 117 & 44,5 & 620 \\
cão & 14.000 & 485 & 34,7 & 745 \\
homem & 65.000 & 1.650 & 25,3 & 920 \\
touro & 600.000 & 12.100 & 20 & 1.890 \\
elefante & 3.672 .000 & 49.000 & 13,4 & 2.060 \\
\hline
\end{tabular}


e assemelhados que não levarem em consideração estes aspectos podem cometer equívocos: trabalhos podem ser criticados, projetos de pesquisa podem ter financiamentos negados, prêmios podem deixar de ser obtidos e igualmente bolsas de estudo perdidas.

Afinal, não existem protocolos definidos sobre dosagens de medicamentos e sua extrapolação do homem para os animais?

Existem!

As áreas do conhecimentos são a Farmacocinética e a Toxicologia, enquanto a metodologia chama-se extrapolação alométrica ${ }^{3,10,11,13,16}$. Existem planilhas nas quais inserimos a espécie, a taxa metabólica e outras características animais, com resultado final de dosagem adequada a ser extrapolada. Esta preocupação $2,9,10,13,16$ de extrapolação de humanos para animais remete-nos ao início do século XX. Este termo vem do Grego állos e significa outro, um outro, diferente ou estranho.

Você já viu esta preocupação e extrapolação alométrica em trabalhos anteriores ${ }^{1,4,5,6,8,14,15,18,19,20}$ sobre testes de medicamentos durante a movimentação dentária induzida? Um exemplo da falta desta metodologia padronizadora e alométrica pode ser obtido ao analisar-se o trabalho de Arias e MarquesOrozco $^{1}$, publicado no Am. J. Orthod Dentofacial Orthop. em 2006, no qual não houve qualquer explicação ou justificativa para as doses utilizadas de aspirina, acetaminofeno e ibuprofeno a que foram submetidos os ratos durante a movimentação dentária induzida. Mesmo em outros modelos inflamatórios em ratos e testando-se o efeito analgésico e antiinflamatório da aspirina não se detecta a preocupação alométrica ou a explicitação do "porque ou como a dosagem e a posologia" foram escolhidas ou determinadas, como no trabalho de LaBuda e Fuchs $^{7}$, em 2001 ?

As agências fomentadoras de pesquisa, juntamente com as universidades, pressionam por número de publicações, sem critérios para avaliar a qualidade das mesmas, apenas consideram a classificação dos veículos de comunicação em que fo- ram aceitos. Assim se criam verdadeiras "correntes literárias", onde um trabalho vai citando outro, e assim sucessivamente, muitas vezes sem checar detalhes metodológicos fundamentais. A simples publicação de um trabalho não lhe dá automaticamente credibilidade, apenas permite sua acessibilidade à comunidade científica. A credibilidade vem com a leitura e análise crítica, mas cada vez menos podem ser caracterizadas como críticas as leituras de trabalhos, quase sempre restritas a resumos e conclusões. Exigir que um determinado trabalho utilize necessariamente as mesmas dosagens, posologias e metodologias de trabalhos anteriores pode ser o caminho mais curto para perpetuar erros metodológicos na concepção de trabalhos científicos sobre movimentação dentária induzida experimental 1,4,5,6,8,14,15,18,19,20.

A literatura sobre os efeitos dos medicamentos durante a movimentação dentária induzida ainda é carente de metodologia padronizada, em todos os seus aspectos. $\mathrm{O}$ modelo murino é o mais utilizado e universalmente aceito como o mais barato, ético e passível de extrapolação para o homem. Podemos até ousar afirmar que, pelo menos, o modelo animal está padronizado: o rato.

No entanto, quando se avalia as dosagens dos medicamentos, o tempo de aplicação e a preparação dos mesmos, a falta de padronização é absoluta ${ }^{1,4,5,6,8,14,15,18,19,20}$. Alguns trabalhos utilizam subdosagens, outros superdosagens. Há uma falta de preocupação ou dificuldade não explicitada em transferir a forma de utilização do medicamento no homem para o animal.

Como exemplo, podemos utilizar os analgésicos e antiinflamatórios. Se no homem a dose de analgésicos é única ou eventual (mesmo que estes medicamentos, com o seu uso prolongado, tenham efeito antiinflamatório), mas se queira avaliar o efeito na movimentação dentária induzida quando são utilizados como analgésicos, deve-se aplicar a dosagem única também no animal ou então dosagem eventual e aleatória, se for o caso. Estes aspectos são considerados na metodologia de extrapolação alométrica. 
Quase todos os analgésicos comercializados e aplicados no homem, em nossos dias, atuam modulando, entre outros mecanismos de ação simultâneos, a síntese de prostaglandinas, interferindo no ciclo do ácido araquidônico. Em uma única dose, a redução de prostaglandinas é transitória, mas suficiente para fazer desaparecer o quadro de dor do paciente durante a movimentação ortodôntica, quando detectado este desconforto. Por sua vez, a redução prolongada de prostaglandinas por estes medicamentos a partir de doses regulares e repetidas, promove um efeito redutor do edema, conhecido como efeito antiinflamatório, muito utilizado nos períodos pós-operatórios cirúrgicos.

Numa situação como em que se queira testar medicamentos em sua ação analgésica, não parece coerente e minimamente razoável que se utilize estes medicamentos com regularidade, em doses antiinflamatórias, durante toda a movimentação dentária induzida. Infelizmente isto ocorre, equivocadamente, em muitos trabalhos na literatura sobre a influência de analgésicos na movimentação dentária induzida $1,4,5,6,8,14,15,18,19,20$. Se estas doses utilizadas nestes trabalhos publicados fossem extrapoladas para o homem, como na grande maioria dos experimentos publicados, os efeitos sobre o movimento ortodôntico seriam desconsiderados, pois os efeitos tóxicos e colaterais no organismo como um todo seriam prioritários: estaríamos frente a uma superdosagem.

Alguém já viu algum trabalho $1,4,5,6,8,14,15,18,19,20$ sobre influência de analgésicos e/ou antiinflamatórios discutir a diferença de doses e posologia a ponto de distinguir se está testando-se o medicamento com indicação de analgesia ou de efeito antiinflamatório? Ou ainda, em algum destes trabalhos ${ }^{1,4,5,6,8,14,15}$, $18,19,20$, procura-se extrapolar, em relação à dosagem, o que seria equivalente para o homem - em termos de quilograma/peso utilizado no trabalho experimental realizado - utilizando-se a metodologia da extrapolação alométrica?

A pesquisa, especialmente em Ortodontia, pode também ser clínica. Um profissional orto- dontista pode, ao longo de um ano, iniciar o tratamento de 20 pacientes que não apresentaram necessidade de ingerir um determinado analgésico. Estes pacientes constituiriam o grupo controle. Outros 20 pacientes com o mesmo tipo de má oclusão, morfologia dentária, mesmo perfil etário e de gênero, submeter-se-iam ao mesmo tipo de tratamento, com o mesmo operador, mas receberiam, por necessidade clínica, o analgésico determinado. Estes pacientes constituiriam o grupo experimental. Provavelmente o grupo experimental apresentaria menor velocidade de movimentação dentária induzida e maior índice de reabsorção radicular após o tratamento. Naturalmente o autor poderia concluir que o medicamento foi o responsável por esta diferença que, até estatisticamente, seria significante. No entanto, a conclusão estaria equivocadíssima: a dor poderia estar relacionada à maior intensidade de força, o que resultaria em menor velocidade de movimentação dentária induzida. As áreas de hialinização e as lesões da camada cementoblástica seriam maiores, resultando em maior índice de reabsorção radicular.

A alternativa para evitar esta interpretação equivocada dos resultados está em administrar analgésicos nos 40 pacientes que indicassem necessidade a partir de sintomatologia dolorosa. Mas 20 destes pacientes receberiam o medicamento na forma de placebo. Porém, nem os pacientes, nem o profissional, saberiam quais os 20 pacientes que estariam recebendo o placebo. Os comprimidos seriam iguais na forma, cor, peso, sabor e embalagem; apenas o farmacêutico saberia informar, depois da obtenção dos dados, quem ingeriu o verdadeiro medicamento. Só então os dados seriam tabulados e os cálculos estatísticos realizados. Esta metodologia chama-se estudo duplo cego: nem paciente nem o operador sabem quem recebeu o tratamento, apenas quando terminada a fase experimental do trabalho essa informação é divulgada.

Uma vez uniformizados os pacientes e o tipo de má oclusão, o planejamento e o tratamento ortodôntico e o método de estudo duplo cego, ficará difícil 
comparar os resultados com os da literatura, pois os trabalhos sobre influência de analgésicos e/ou antiinflamatórios concebidos com estas preocupações metodológicas praticamente inexistem.

A pesquisa aplicada quase sempre busca avaliar, resolver ou esclarecer uma questão específica, um problema, uma situação determinada, uma dúvida, uma técnica. A idéia advém de um problema ou situação clínica, no caso da Ortodontia. Um último questionamento, assim, torna-se inevitável: quem teve problemas na velocidade de movimentação ortodôntica e no índice de reabsorções em sua prática clínica, quando os pacientes utilizam analgésicos para amenizar a dor e desconforto após a ativação dos aparelhos? Este problema existe?

Não! O problema, por mais incrivel que possa parecer, está no fato de alguns ortodontistas não indicarem, desaconselharem e até proibirem o paciente de ingerir analgésicos frente ao desconforto

\section{REFERÊNCIAS}

1. ARIAS, O. R.; MÁRQUEZ-OROZCO, M. C. Aspirin, acetaminophen, and ibuprofen: their effects on orthodontic tooth movement. Am. J. Orthod. Dentofacial Orthop., St. Louis, v. 130, no. 5, p. 364-370, Sept. 2006.

2. BENEDICT, F. G. Vital energetics: a study in comparative basal metabolism. Washington: Carnegie Inst. Publ. 1938. p. 1-215.

3. BRITO, H. F. V. Determinação da taxa metabólica basal em Dasyprocta azarae por calorimetria indireta. 2004. Dissertação (Mestrado)-Universidade Federal do Paraná, Curitiba, 2004

4. CHUMBLEY, A. B.; TUNCAY, O. C. The effect of indomethacin (an aspirin-like drug) on the rate of orthodontic tooth movement. Am. J. Orthod., St. Louis, v. 89, no. 4, p. 312-314, Apr. 1986.

5. GIUNTA, D. et al. Influence of indomethacin on bone turnover related to orthodontic tooth movement in miniature pigs. Am. J. Orthod. Dentofacial Orthop., St. Louis, v. 108, no. 4, p. 361-266, Oct. 1995.

6. KEHOE, M. J. et al. The effect of acetaminophen, ibuprofen, and misoprostol on prostanglandin E2 synthesis and the degree and rate of orthodontic tooth movement. Angle Orthod., Appleton, v. 66, p. 339-350, Apr. 1995.

7. LABUDA, C. J.; FUCHS, P. N. Low dose aspirin attenuates escape/avoidance behavior, but does not reduce mechanical hyperalgesia in a rodent model of inflammatory pain. Neurosci. Lett, Amsterdam, v. 300, p. 137-140, 2001.

8. LAW, S. L. S. et al. An evaluation of preoperative ibuprofen for treatment of pain associated with orthodontic separator placement. Am. J. Orthod. Dentofacial Orthop., St. Louis, v. 118, no. 6, p. 629-635, Dec. 2000.

9. LIN, J. H. Applications and limitations of interspecies scaling and in vitro extrapolation in pharmacokinetics. Drug Metab. Dispos., Baltimore, v. 26, p. 1202-1212, 1998.

10. MAHMOOD, I. Allometric issues in drug development. J. Pharm. Sci., New York, v. 88, p. 1101-1106, 1999. ou dor provocados logo depois de algumas horas de ativação dos aparelhos! Só pode ser por simples crença ou dogma, próprios dos preceitos religiosos, mas não fundamentados em evidências metodológicas próprias da atividade científica.

As perguntas e questionamentos que fizemos são apenas pontos de partida para induzir reflexões e necessariamente são questionáveis e não absolutamente corretas. A finalidade principal de um insight está em inquietar para fazer progredir, melhorar e aperfeiçoar nossa querida Ortodontia.

Quanto à pesquisa sobre o efeito de analgésicos e antiinflamatórios na movimentação dentária induzida, apenas nos resta concluir que os cálculos devem ser refeitos à luz da extrapolação alométrica e, somente após este recálculo, considerar os resultados válidos ou não, pois é muito alta a possibilidade das doses e posologias serem inadequadas e os resultados decorrentes altamente questionáveis?
11. PACHALY, J. R.; BRITO, H. F. V. Emprego do método de extrapolação alométrica no cálculo de protocolos posológicos para animais selvagens. A Hora Veterinária, Porto Alegre, v. 20, n. 118, p. 59-65, 2000

12. PICHOTKA, J. Funciones metabolicas de los organismos. In: KEIDEL, W. D. Fisiologia. Barcelona: Salvat, 1971. p. 154207.

13. RITSCHEL, W. A.; VACHHARAJANI, N. N.; JOHNSON, R. D.; HUSSAIN, A. S. The allometric approach for interspecies scaling of pharmacokinetic parameters. Comp. Biochem. Physiol., Oxford, v. 103, p. 249-253, 1992.

14. ROCHE, J. J.; CISNEROS, G. J.; ACS, G. The effect of acetaminophen on tooth movement in rabbits. Angle Orthod., Appleton, v. 67, no. 3, 231-236, 1997.

15. SARI, E.; OLMEZ, H.; GURTON, U. Comparison of some effects of acetylsalicylic acid and rofecoxib during orthodontic tooth movement. Am. J. Orthod. Dentofacial Orthop., St. Louis, v. 125, no. 3, p. 310-315, Mar. 2004.

16. SCHNEIDER, K.; OLTMANNS, J.; HASSAUER, M. Allometric principles for interspecies extrapolation in toxicological risk assessment: empirical investigations. Regul. Toxicol. Pharmacol., New York, v. 39, p. 334-347, 2004.

17. WAHRLICH, V.; ANJOS, L. A. Aspectos históricos e metodológicos da medição e estimativa da taxa metabólica basal: uma revisão da literatura. Cad. Saúde Pública, Rio de Janeiro, v. 17, n. 4, p. 801-817, jul./ago. 2001.

18. WALKER, J. B.; BURING, S. M. NSAID impairment of orthodontic tooth movement. Ann. Pharmacother., Cincinnati, v. 35, p. 113-115, Jan. 2001.

19. WONG, A.; REYNOLDS, E. C.; WEST, V. C. The effect of acetylsalicylic acid on orthodontic tooth movement in the Guinea pig. Am. J. Orthod. Dentofacial Orthop., St. Louis, v. 102, no. 4, 360-365, Oct. 1992.

20. ZHOU, D.; HUGHES, B.; KING, J. Histomorphometric and biochemical study of osteoclasts at orthodontic compression sites in the rat during indomethacin inhibition. Arch. Oral Biol., Oxford, v. 42, no. 10/11, p. 717-726, 1997. 